\title{
Ring configurations of point vortices in polar atmospheres
}

\author{
David G. Dritschel ${ }^{1 *}$ \\ ${ }^{1}$ Mathematical Institute, University of St Andrews \\ North Haugh, St Andrews KY16 9SS, UK \\ Received February 1, 2021; accepted Month XX, 20XX
}

\begin{abstract}
This paper examines the stability and nonlinear evolution of configurations of equalstrength point vortices equally spaced on a ring of constant radius, with or without a central vortex, in the three-dimensional quasi-geostrophic compressible atmosphere model. While the ring lies at constant height, the central vortex can be at a different height and also have a different strength to the vortices on the ring. All such configurations are relative equilibria, in the sense that they steadily rotate about the $z$ axis. Here, the domains of stability for two or more vortices on a ring with an additional central vortex are determined. For a compressible atmosphere, the problem also depends on the density scale height $H$, the vertical scale over which the background density varies by a factor $e$. Decreasing $H$ while holding other parameters fixed generally stabilises a configuration. Nonlinear simulations of the dynamics verifies the linear analysis and reveals potentially chaotic dynamics for configurations having four or more vortices in total. The simulations also reveal the existence of staggered ring configurations, and oscillations between single and double ring configurations. The results are consistent with the observations of ring configurations of polar vortices seen at both of Jupiter's poles [1]
\end{abstract}

MSC2010 numbers: MSC2010

DOI: $10.0000 / \mathrm{S} 1560354700000012$

Keywords: vortex dynamics, point vortices

\section{INTRODUCTION}

The study of the stability of $n$ equally-spaced and identical point vortices lying on a ring goes back to J.J. Thomson in 1883 [14], who examined the two-dimensional (barotropic) case without a central vortex. Thomson found that $n>8$ vortices are linearly unstable (though he erroneously concluded $n=7$ vortices are unstable as well due to numerical inaccuracies). Nearly a century later, Morikawa and Svenson [8] examined "geostrophic" vortices in the single-layer, quasi-geostrophic shallow-water (QGSW) model, and included a central vortex. The QGSW model includes an additional parameter, the Rossby deformation length $L_{D}$. In the limit $L_{D} \rightarrow \infty$, the QGSW model reduces to the barotropic model (or the two-dimensional Euler equations) originally examined by Thomson. Further analysis of the geostrophic system, including nonlinear stability, was presented by Kurakin and Ostrovskaya [7], wherein one may find an excellent literature review of Thomson's problem and its variations.

Recently, Reinaud [10] generalised the study of [8] to the three-dimensional quasi-geostrophic (3DQG) model, using the Boussinesq approximation relevant to oceanic dynamics. Reinaud considered the simplest case for which both the Coriolis and buoyancy frequencies, $f$ and $N$, are constant. Using the rescaled height coordinate $\tilde{z}=N z / f$, the streamfunction $\psi$ is then obtained by inverting Laplace's operator $\nabla^{2}$ (in the rescaled coordinates) on the potential vorticity $q$, taken to be localised at discrete points. The resulting point vortex model is closely analogous to that derived in two dimensions, and indeed the only difference is the form of the Green function.

Here, we extend [10] to the compressible-atmosphere 3DQG model. In this case, the Green function is anisotropic in $z$ due to the exponential decay of the background density profile [12]. Moreover, there is an additional length scale to consider, the $e$-folding length $H$ of density. This

\footnotetext{
${ }^{*}$ E-mail: david.dritschel@st-andrews.ac.uk
} 
makes the problem considerably richer, and introduces another stabilising mechanism for ring configurations of vortices.

The plan of the paper is as follows. The next section reviews the QG model and derives the nonlinear equations for a general system of point vortices. These equations are next linearised in section 3 and cast into an eigenvalue problem to determine the modes of oscillation or stability. Results are presented for a wide range of parameters. The nonlinear behaviour of selected instabilities is examined in section 4. Conclusions are offered in section 5, including a discussion of the relevance to Jupiter's polar vortices [1].

\section{MATHEMATICAL PRELIMINARIES}

\subsection{The Compressible Quasi-Geostrophic Model}

We consider a flow in an unbounded domain (or far from any boundaries). We assume that the flow is shallow, in the sense that horizontal scales are much larger than vertical ones, in order to make the hydrostatic approximation (wherein the vertical acceleration is neglected). Furthermore, we assume that the flow is sufficiently rapidly rotating and strongly stratified to make the geostrophic approximation (wherein the horizontal acceleration is neglected). This requires that both the Rossby number Ro and the Froude number Fr are small compared to unity, specifically $\mathrm{Fr}^{2} \ll \mathrm{Ro}_{\mathrm{O}} \ll 1$. Under these conditions, the Quasi-Geostrophic (QG) model emerges at leading order in Ro [15]. The Rossby number Ro is the ratio of a characteristic vertical vorticity to the background 'planetary vorticity' or Coriolis frequency $f_{0}$. The Froude number Fr, similarly, is the ratio of a characteristic horizontal vorticity to the background Brunt-Väisälä or buoyancy frequency $N_{0}$.

For a compressible atmosphere governed by the ideal gas law and conservation of entropy (or potential temperature), the vertical hydrostatic balance implies that the background density $\rho_{0}(z)$ has an exponential dependence on 'height' $z$, proportional to log pressure, i.e. $\rho_{0}(z)=\rho_{00} e^{-z / H}$ $[2,3]$. Here $H$ is the 'density scale height' (proportional to a reference temperature), and $\rho_{00}$ is a constant reference density (see section 2(a) in [3] for details). Then, for an inviscid adiabatic flow, the unique prognostic equation in the $\mathrm{QG}$ model expresses material conservation of $\mathrm{QG}$ potential vorticity $(\mathrm{PV})$,

$$
\frac{\mathrm{D} q}{\mathrm{D} t} \equiv \frac{\partial q}{\partial t}+u \frac{\partial q}{\partial x}+v \frac{\partial q}{\partial y}=0,
$$

i.e. $q$ is conserved following fluid particles transported by the layerwise-two-dimensional flow $(u(x, y, z, t), v(x, y, z, t))$. The vertical velocity $w$ is higher order in Rossby number Ro and does not directly contribute to PV advection. The horizontal velocity field is determined by 'PV inversion', namely by solving for the streamfunction $\psi$ from the definition of PV,

$$
q=\frac{\partial^{2} \psi}{\partial x^{2}}+\frac{\partial^{2} \psi}{\partial y^{2}}+\frac{1}{\rho_{0}} \frac{\partial}{\partial z}\left(\rho_{0} \frac{f_{0}^{2}}{N_{0}^{2}} \frac{\partial \psi}{\partial z}\right)
$$

then differentiating,

$$
u=-\frac{\partial \psi}{\partial y}, \quad v=\frac{\partial \psi}{\partial x} .
$$

Note, the horizontal velocity field is incompressible (to leading order in Ro). Moreover, PV is transported on constant $z$ surfaces - this is why the motion is considered 'layerwise-twodimensional'.

For an isothermal atmosphere, assumed henceforth, the buoyancy frequency $N_{0}$ is a constant [3]. In this case, the ratio $f_{0} / N_{0}$ can be absorbed into the height coordinate, i.e. replacing $N_{0} z / f_{0}$ by $z$ in Eq. (2.2), to remove any explicit dependence on either $f_{0}$ or $N_{0}$. Typically, this corresponds to a stretch of the vertical coordinate, so that pancake-like structures become more isotropic after scaling [9]. This stretch must also be done for the density scale height $H$, i.e. we also replace $N_{0} H / f_{0}$ by $H$ in $\rho_{0}(z)=\rho_{00} e^{-z / H}$. The simultaneous scaling of $z$ and $H$ leaves the form of $\rho_{0}(z)$ unchanged. 
With these modifications, the definition of PV reduces to a (modified) Helmholtz equation,

$$
\nabla^{2} \psi-\frac{1}{H} \frac{\partial \psi}{\partial z}=q
$$

where $\nabla^{2}$ is the three-dimensional Laplacian. In an infinite domain, the solution for $\psi$ can be found in terms of a Green function $G\left(\boldsymbol{x}^{\prime} ; \boldsymbol{x}\right)$, where $\boldsymbol{x}^{\prime}=\left(x^{\prime}, y^{\prime}, z^{\prime}\right)$ is the 'source point' and $\boldsymbol{x}=(x, y, z)$ is the 'target point', namely

$$
\psi(\boldsymbol{x}, t)=\iiint q\left(\boldsymbol{x}^{\prime}, t\right) G\left(\boldsymbol{x}^{\prime} ; \boldsymbol{x}\right) \mathrm{d} x^{\prime} \mathrm{d} y^{\prime} \mathrm{d} z^{\prime} .
$$

In the limit $H \rightarrow \infty$ (applicable to a Boussinesq, ocean-like fluid), $G$ reduces to the well known form $G=-1 /(4 \pi r)$ where $r=\left\|\boldsymbol{x}^{\prime}-\boldsymbol{x}\right\|$ is the three-dimensional distance between $\boldsymbol{x}^{\prime}$ and $\boldsymbol{x}$. In this case, $G$ is isotropic. However, for finite $H$, this is no longer the case and instead $G$ assumes the form

$$
G\left(\boldsymbol{x}^{\prime} ; \boldsymbol{x}\right)=-\frac{e^{-\left(r+z^{\prime}-z\right) / 2 H}}{4 \pi r},
$$

first derived in [12]. In this case, $G$ is the product of a function of $z^{\prime}-z$ and a function of $r$.

\subsection{The Point Vortex Model}

The Green-function formulation above is particularly convenient for deriving the equations governing the motion of an arbitrary number $n$ of point singularities of PV located at $\boldsymbol{x}_{j}(t)$, for $j=1, \ldots, n$. Then, the distribution of PV is represented by a sum of Dirac measures (or $\delta$ 'functions'),

$$
q\left(\boldsymbol{x}^{\prime}, t\right)=4 \pi \sum_{j=1}^{n} \kappa_{j} \delta\left(\boldsymbol{x}^{\prime}-\boldsymbol{x}_{j}(t)\right),
$$

where $\kappa_{j}$ is the 'strength' of vortex $j$, equal to the volume integral of its PV divided by $4 \pi$. Substituting Eq. (2.7) into Eq. (2.5), the streamfunction $\psi(\boldsymbol{x}, t)$ at any point $\boldsymbol{x}$, except at a point vortex position, reduces to the sum

$$
\psi(\boldsymbol{x}, t)=\sum_{j=1}^{n} \kappa_{j} e^{\left(z-z_{j}\right) / 2 H} g\left(\Delta_{j}\right), \quad \text { where } \quad g(r) \equiv-\frac{e^{-r / 2 H}}{r}
$$

and $\Delta_{j}=\left\|\boldsymbol{x}-\boldsymbol{x}_{j}(t)\right\|$.

From Eq. (2.8), the layerwise-two-dimensional velocity field follows upon differentiation:

$$
\begin{aligned}
& u(\boldsymbol{x}, t)=-\sum_{j=1}^{n} \kappa_{j} e^{\left(z-z_{j}\right) / 2 H} \xi\left(\Delta_{j}\right)\left(y-y_{j}\right), \\
& v(\boldsymbol{x}, t)=+\sum_{j=1}^{n} \kappa_{j} e^{\left(z-z_{j}\right) / 2 H} \xi\left(\Delta_{j}\right)\left(x-x_{j}\right)
\end{aligned}
$$

where

$$
\xi(r) \equiv \frac{1}{r} \frac{\mathrm{d} g}{\mathrm{~d} r}=\frac{1}{r^{2}}\left(\frac{1}{2 H}+\frac{1}{r}\right) e^{-r / 2 H} .
$$

Again, Eqs. (2.9) and (2.10) are valid everywhere except at a vortex position. There is no selfinduced vortex motion on account of symmetry $[4,6]$, so when $\boldsymbol{x}=\boldsymbol{x}_{k}(t)$, the index $j=k$ is excluded from the sums above.

The vortex motion then follows from

$$
\dot{x}_{k}=u\left(\boldsymbol{x}_{k}, t\right)=-\sum_{j=1, j \neq k}^{n} \kappa_{j} e^{\left(z_{k}-z_{j}\right) / 2 H} \xi\left(\Delta_{k j}\right)\left(y_{k}-y_{j}\right),
$$




$$
\dot{y}_{k}=v\left(\boldsymbol{x}_{k}, t\right)=+\sum_{j=1, j \neq k}^{n} \kappa_{j} e^{\left(z_{k}-z_{j}\right) / 2 H} \xi\left(\Delta_{k j}\right)\left(x_{k}-x_{j}\right)
$$

where $\Delta_{k j}=\left\|\boldsymbol{x}_{k}(t)-\boldsymbol{x}_{j}(t)\right\|$ is the three-dimensional distance between vortices $j$ and $k$, and a dot stands for an ordinary time derivative. Note, $\dot{z}_{k}=0$ as there is no vertical motion: each vortex remains at a constant height $z_{k}$ for all time.

\section{LINEAR DYNAMICS AND STABILITY}

\subsection{The Basic State}

We consider a ring configuration of $n$ equal strength vortices equally spaced on the unit circle in the $z=0$ plane, together with a central vortex of generally different strength lying along the $z$ axis at $z=z_{0}$. It is convenient to use the index 0 for the central vortex, so its strength is $\kappa_{0}$ and its undisturbed position is $x_{0}=y_{0}=0$. The vortices on the ring are indexed $j=1, \ldots, n$, have strengths $\kappa_{j}=1$ (without loss of generality), radii $R_{j}=1$, azimuthal angles $\phi_{j}=\bar{\phi}_{j} \equiv 2 \pi(j-1) / n$, and heights $z_{j}=0$. By symmetry, this is a relative equilibrium, in the sense that it steadily rotates at a fixed rate $\Omega$.

The rotation rate $\Omega$ equals the $y$ velocity component $v_{1}$ of the first vortex lying at $x_{1}=1$ and $y_{1}=0$. Therefore, applying Eq. (2.13) for $k=1$ and extending the sum from $j=0$ to $n$, we find

$$
\Omega=\kappa \xi_{0}+2 \sum_{j=2}^{n} \xi_{j} s_{j}^{2}
$$

where $\kappa \equiv \kappa_{0} e^{-z_{0} / 2 H}, \xi_{j} \equiv \xi\left(\bar{r}_{j}\right)$,

$$
\bar{r}_{0}=\sqrt{1+z_{0}^{2}}, \quad \bar{r}_{j}=\sqrt{2\left(1-\cos \bar{\phi}_{j}\right)}=2 s_{j} \quad(j>0),
$$

and finally

$$
s_{j}=\sin \frac{1}{2} \bar{\phi}_{j}, \quad c_{j}=\cos \frac{1}{2} \bar{\phi}_{j} .
$$

In Eq. (3.1), the $2 s_{j}^{2}$ term comes from rewriting $1-x_{j}=1-\cos \bar{\phi}_{j}$ as in Eq. (3.2).

\subsection{The Linearised Equations}

It is natural to use polar coordinates, $R_{j}(t)$ and $\phi_{j}(t)$, for each vortex on the ring, and to use Cartesian coordinates $x_{0}(t)$ and $y_{0}(t)$ for the central vortex. The $z$ coordinates of the vortices do not change.

From Eqs. (2.12) and (2.13), the central vortex $k=0$ evolves according to

$$
\dot{x}_{0}=-e^{z_{0} / 2 H} \sum_{j=1}^{n} \xi\left(\Delta_{0 j}\right)\left(y_{0}-y_{j}\right), \quad \dot{y}_{0}=e^{z_{0} / 2 H} \sum_{j=1}^{n} \xi\left(\Delta_{0 j}\right)\left(x_{0}-x_{j}\right) .
$$

Meanwhile vortex $j=1$ on the ring evolves according to

$$
\dot{R}_{1}=-\left.\frac{1}{R_{1}} \frac{\partial \psi_{1}}{\partial \phi}\right|_{\boldsymbol{x}=\boldsymbol{x}_{1}}, \quad \dot{\phi}_{1}=\left.\frac{1}{R_{1}} \frac{\partial \psi_{1}}{\partial R}\right|_{\boldsymbol{x}=\boldsymbol{x}_{1}}
$$

where $\psi_{1}(\boldsymbol{x}, t)$ is the streamfunction excluding $j=1$ in Eq. (2.8) (extended from $j=0$ to $n$ ). Using $r_{j} \equiv \Delta_{1 j}=\left\|\boldsymbol{x}_{1}-\boldsymbol{x}_{j}\right\|$ to simplify notation, we have

$$
\begin{aligned}
& \dot{R}_{1}=\kappa \xi\left(r_{0}\right)\left(y_{0} \cos \phi_{1}-x_{0} \sin \phi_{1}\right)+\sum_{j=2}^{n} \xi\left(r_{j}\right) R_{j} \sin \left(\phi_{j}-\phi_{1}\right) \\
& \dot{\phi}_{1}=\kappa \xi\left(r_{0}\right)\left(1-R_{1}^{-1}\left(x_{0} \cos \phi_{1}+y_{0} \sin \phi_{1}\right)\right)+\sum_{j=2}^{n} \xi\left(r_{j}\right)\left(1-R_{1}^{-1} R_{j} \cos \left(\phi_{j}-\phi_{1}\right)\right) .
\end{aligned}
$$


The equations for the other vortices on the ring follow by symmetry.

It is useful to adopt a reference frame rotating at the rate $\Omega$ so that the undisturbed vortex configuration is stationary. This amounts to adding $(\Omega y,-\Omega x)$ to the horizontal velocity $(u, v)$, or subtracting $\Omega$ from $\dot{\phi}_{j}$. Consider sufficiently small disturbances

$$
\left|x_{0}\right| \ll 1, \quad\left|y_{0}\right| \ll 1, \quad R_{j}^{\prime}=R_{j}-1 \ll 1 \quad \text { and } \quad \phi_{j}^{\prime}=\phi_{j}-\bar{\phi}_{j} \ll 1
$$

so that the linearised equations of motion accurately approximate the dynamics, at least over a finite time interval.

For the central vortex, indexed 0 , note that

$$
\Delta_{0 j}=\left\|\boldsymbol{x}_{0}-\boldsymbol{x}_{j}\right\| \approx \bar{r}_{0}+r_{j}^{\prime} \quad \text { with } \quad r_{j}^{\prime}=\frac{R_{j}^{\prime}-x_{0} \cos \bar{\phi}_{j}-y_{0} \sin \bar{\phi}_{j}}{\bar{r}_{0}}
$$

to first order $\left(\bar{r}_{0}\right.$ is defined in Eq. (3.2)), and hence

$$
\xi\left(\Delta_{0 j}\right) \approx \xi\left(\bar{r}_{0}\right)+\frac{\mathrm{d} \xi}{\mathrm{d} r}\left(\bar{r}_{0}\right) r_{j}^{\prime}=\xi_{0}+\mu_{0} r_{j}^{\prime}
$$

where, for any $j$,

$$
\mu_{j}=\mu\left(\bar{r}_{j}\right), \quad \text { with } \quad \mu(r) \equiv \frac{\mathrm{d} \xi}{\mathrm{d} r}=-\left(\frac{1}{4 H^{2} r^{2}}+\frac{3}{2 H r^{3}}+\frac{3}{r^{4}}\right) e^{-r / 2 H} .
$$

Moreover,

$$
\begin{aligned}
& R_{j} \sin \phi_{j} \approx \sin \bar{\phi}_{j}+R_{j}^{\prime} \sin \bar{\phi}_{j}+\phi_{j}^{\prime} \cos \bar{\phi}_{j} \\
& R_{j} \cos \phi_{j} \approx \cos \bar{\phi}_{j}+R_{j}^{\prime} \cos \bar{\phi}_{j}-\phi_{j}^{\prime} \sin \bar{\phi}_{j} .
\end{aligned}
$$

Using these results, and $e_{0} \equiv e^{z_{0} / 2 H}$, the central vortex satisfies to first order in perturbations

$$
\begin{aligned}
& \dot{x}_{0}=\Omega y_{0}-e_{0} \sum_{j=1}^{n} \xi_{0}\left(y_{0}-R_{j}^{\prime} \sin \bar{\phi}_{j}-\phi_{j}^{\prime} \cos \bar{\phi}_{j}\right)-\frac{\mu_{0}}{\bar{r}_{0}} \sin \bar{\phi}_{j}\left(R_{j}^{\prime}-x_{0} \cos \bar{\phi}_{j}-y_{0} \sin \bar{\phi}_{j}\right) \\
& \dot{y}_{0}=-\Omega x_{0}+e_{0} \sum_{j=1}^{n} \xi_{0}\left(x_{0}-R_{j}^{\prime} \cos \bar{\phi}_{j}+\phi_{j}^{\prime} \sin \bar{\phi}_{j}\right)-\frac{\mu_{0}}{\bar{r}_{0}} \cos \bar{\phi}_{j}\left(R_{j}^{\prime}-x_{0} \cos \bar{\phi}_{j}-y_{0} \sin \bar{\phi}_{j}\right) .
\end{aligned}
$$

These can be simplified using

$$
\sum_{j=1}^{n} \sin \bar{\phi}_{j} \cos \bar{\phi}_{j}=0, \quad \sum_{j=1}^{n} \sin ^{2} \bar{\phi}_{j}=\frac{n}{2}-\delta_{n 2} \quad \text { and } \quad \sum_{j=1}^{n} \cos ^{2} \bar{\phi}_{j}=\frac{n}{2}+\delta_{n 2},
$$

where $\delta_{i j}=1$ if $i=j$ and 0 otherwise, to

$$
\begin{aligned}
& \dot{x}_{0}=-P_{0 n} y_{0}+\gamma_{0} e_{0} \sum_{j=1}^{n} R_{j}^{\prime} \sin \bar{\phi}_{j}+\xi_{0} e_{0} \sum_{j=1}^{n} \phi_{j}^{\prime} \cos \bar{\phi}_{j} \\
& \dot{y}_{0}=Q_{0 n} x_{0}-\gamma_{0} e_{0} \sum_{j=1}^{n} R_{j}^{\prime} \cos \bar{\phi}_{j}+\xi_{0} e_{0} \sum_{j=1}^{n} \phi_{j}^{\prime} \sin \bar{\phi}_{j}
\end{aligned}
$$

where

$$
\gamma_{0} \equiv \xi_{0}+\frac{\mu_{0}}{\bar{r}_{0}}
$$

and

$$
\begin{gathered}
P_{0 n} \equiv e_{0}\left[n \xi_{0}+\frac{\mu_{0}}{\bar{r}_{0}}\left(\frac{n}{2}-\delta_{n 2}\right)\right]-\Omega \\
Q_{0 n} \equiv e_{0}\left[n \xi_{0}+\frac{\mu_{0}}{\bar{r}_{0}}\left(\frac{n}{2}+\delta_{n 2}\right)\right]-\Omega .
\end{gathered}
$$

REGULAR AND CHAOTIC DYNAMICS Vol. 00 No. $0 \quad 0000$ 
Next consider the first vortex $j=1$ on the ring. Here,

$$
r_{j}=\left\|\boldsymbol{x}_{j}-\boldsymbol{x}_{1}\right\| \approx \bar{r}_{j}+r_{j}^{\prime} \quad \text { with } \quad r_{j}^{\prime}=\left(R_{j}^{\prime}+R_{1}^{\prime}\right) s_{j}+\left(\phi_{j}^{\prime}-\phi_{1}^{\prime}\right) c_{j},
$$

while

$$
r_{0}=\left\|\boldsymbol{x}_{0}-\boldsymbol{x}_{1}\right\| \approx \bar{r}_{0}+r_{0}^{\prime} \quad \text { with } \quad r_{0}^{\prime}=\frac{R_{1}^{\prime}-x_{0}}{\bar{r}_{0}}
$$

Moreover,

$$
\begin{aligned}
R_{j} \sin \left(\phi_{j}-\phi_{1}\right) & \approx \sin \bar{\phi}_{j}+R_{j}^{\prime} \sin \bar{\phi}_{j}+\left(\phi_{j}^{\prime}-\phi_{1}^{\prime}\right) \cos \bar{\phi}_{j} \\
1-R_{1}^{-1} R_{j} \cos \left(\phi_{j}-\phi_{1}\right) & \approx 1-\cos \bar{\phi}_{j}-\left(R_{j}^{\prime}-R_{1}^{\prime}\right) \cos \bar{\phi}_{j}+\left(\phi_{j}^{\prime}-\phi_{1}^{\prime}\right) \sin \bar{\phi}_{j} .
\end{aligned}
$$

Using these results, the first vortex on the ring satisfies to first order in perturbations

$$
\begin{gathered}
\dot{R}_{1}^{\prime}=\kappa \xi_{0} y_{0}+\sum_{j=2}^{n} \xi_{j}\left[R_{j}^{\prime} \sin \bar{\phi}_{j}+\left(\phi_{j}^{\prime}-\phi_{1}^{\prime}\right) \cos \bar{\phi}_{j}\right] \\
+\mu_{j} \sin \bar{\phi}_{j}\left[\left(R_{j}^{\prime}+R_{1}^{\prime}\right) s_{j}+\left(\phi_{j}^{\prime}-\phi_{1}^{\prime}\right) c_{j}\right] \\
\dot{\phi}_{1}^{\prime}=\kappa\left(\frac{\mu_{0}}{\bar{r}_{0}} R_{1}^{\prime}-\gamma_{0} x_{0}\right)+\sum_{j=2}^{n} \xi_{j}\left[-\left(R_{j}^{\prime}-R_{1}^{\prime}\right) \cos \bar{\phi}_{j}+\left(\phi_{j}^{\prime}-\phi_{1}^{\prime}\right) \sin \bar{\phi}_{j}\right] \\
+\mu_{j}\left(1-\cos \bar{\phi}_{j}\right)\left[\left(R_{j}^{\prime}+R_{1}^{\prime}\right) s_{j}+\left(\phi_{j}^{\prime}-\phi_{1}^{\prime}\right) c_{j}\right],
\end{gathered}
$$

where $\mu_{j}$ is defined in Eq. (3.11). By symmetry, the term involving $R_{1}^{\prime}$ on the second line of the $\dot{R}_{1}^{\prime}$ equation vanishes, as do all terms involving $\phi_{1}^{\prime}$ in the $\dot{\phi}_{1}^{\prime}$ equation. Using $\sin \bar{\phi}_{j}=2 s_{j} c_{j}$ and $\cos \bar{\phi}_{j}=2 c_{j}^{2}-1$ allows us to simplify the above equations to

$$
\begin{aligned}
& \dot{R}_{1}^{\prime}=\kappa \xi_{0} y_{0}+\sum_{j=2}^{n} \eta_{j} s_{j} c_{j} R_{j}^{\prime}+\left(\eta_{j} c_{j}^{2}-\xi_{j}\right)\left(\phi_{j}^{\prime}-\phi_{1}^{\prime}\right), \quad \text { where } \eta_{j} \equiv 2\left(\mu_{j} s_{j}+\xi_{j}\right), \\
& \dot{\phi}_{1}^{\prime}=-\kappa \gamma_{0} x_{0}+R_{1}^{\prime}\left(\kappa \frac{\mu_{0}}{\bar{r}_{0}}+\sum_{j=2}^{n} \xi_{j}+2 s_{j}^{2}\left(\mu_{j} s_{j}-\xi_{j}\right)\right)+\sum_{j=2}^{n}\left(\eta_{j} s_{j}^{2}-\xi_{j}\right) R_{j}^{\prime}+\eta_{j} s_{j} c_{j} \phi_{j}^{\prime} .
\end{aligned}
$$

For the other vortices $(k>1)$ on the ring, the equations follow by a simple rotation through $\bar{\phi}_{k}$ :

$$
\begin{aligned}
\dot{R}_{k}^{\prime}=\kappa \xi_{0}\left(y_{0} \cos \bar{\phi}_{k}-x_{0} \sin \bar{\phi}_{k}\right) & +\sum_{j=2}^{n} \eta_{j} s_{j} c_{j} R_{m(j+k)}^{\prime}+\left(\eta_{j} c_{j}^{2}-\xi_{j}\right)\left(\phi_{m(j+k)}^{\prime}-\phi_{k}^{\prime}\right), \\
\dot{\phi}_{k}^{\prime}=-\kappa \gamma_{0}\left(x_{0} \cos \bar{\phi}_{k}+y_{0} \sin \bar{\phi}_{k}\right) & +R_{k}^{\prime}\left(\kappa \frac{\mu_{0}}{\bar{r}_{0}}+\sum_{j=2}^{n} \xi_{j}+2 s_{j}^{2}\left(\mu_{j} s_{j}-\xi_{j}\right)\right) \\
& +\sum_{j=2}^{n}\left(\eta_{j} s_{j}^{2}-\xi_{j}\right) R_{m(j+k)}^{\prime}+\eta_{j} s_{j} c_{j} \phi_{m(j+k)}^{\prime},
\end{aligned}
$$

where $m(j+k) \equiv \bmod (j+k-2, n)+1$ (this reduces to $j$ when $k=1)$.

\subsection{The Eigenvalue Problem}

We next seek normal-mode or 'eigen' solutions of the form

$$
x_{0}=\hat{x} e^{\sigma t}, \quad y_{0}=\hat{y} e^{\sigma t}, \quad R_{j}^{\prime}=\hat{R} e^{\mathrm{i} m \bar{\phi}_{j}} e^{\sigma t} \quad \text { and } \quad \phi_{j}^{\prime}=\hat{\phi} e^{\mathrm{i} m \bar{\phi}_{j}} e^{\sigma t},
$$

where $\sigma$ is the growth rate (or eigenvalue, generally complex), and the 'mode' index $m$ ranges from 1 to $n-1$. This form is a consequence of the symmetry of the basic state (see e.g. [4]). In fact, only modes $m=1$ to $[n / 2]$, where [.] denotes 'integer value of', are unique. Mode $m=0$ 
and its complement $m=n$ are excluded as they violate conservation of angular momentum (they correspond to a different equilibrium state).

Inserting the perturbation forms in Eq. (3.33) into equations Eqs. (3.17), (3.18), (3.28), (3.29), and using

$$
\begin{aligned}
& \hat{y} \cos \bar{\phi}_{k}-\hat{x} \sin \bar{\phi}_{k}=\frac{1}{2}(\hat{y}+\mathrm{i} \hat{x}) e^{\mathrm{i} \bar{\phi}_{k}}+\frac{1}{2}(\hat{y}-\mathrm{i} \hat{x}) e^{-\mathrm{i} \bar{\phi}_{k}} \\
& \hat{x} \cos \bar{\phi}_{k}+\hat{y} \sin \bar{\phi}_{k}=\frac{1}{2}(\hat{x}-\mathrm{i} \hat{y}) e^{\mathrm{i} \bar{\phi}_{k}}+\frac{1}{2}(\hat{x}+\mathrm{i} \hat{y}) e^{-\mathrm{i} \bar{\phi}_{k}}
\end{aligned}
$$

results in the following algebraic system of equations for $\hat{x}, \hat{y}, \hat{R}$ and $\hat{\phi}$ :

$$
\begin{aligned}
\sigma \hat{x} & =-P_{0 n} \hat{y}+\mathrm{i} \gamma_{0} S_{m n} \hat{R}+\xi_{0} C_{m n} \hat{\phi} \\
\sigma \hat{y} & =Q_{0 n} \hat{x}-\gamma_{0} C_{m n} \hat{R}+\mathrm{i} \xi_{0} S_{m n} \hat{\phi} \\
\sigma \hat{R} & =\frac{\kappa \xi_{0}}{2}(\hat{y}+\mathrm{i} \hat{x}) e^{-\mathrm{i}(m-1) \bar{\phi}_{k}}+\frac{\kappa \xi_{0}}{2}(\hat{y}-\mathrm{i} \hat{x}) e^{-\mathrm{i}(m+1) \bar{\phi}_{k}}+\mathrm{i} \tilde{a}_{m} \hat{R}+\tilde{c}_{m} \hat{\phi} \\
\sigma \hat{\phi} & =-\frac{\kappa \gamma_{0}}{2}(\hat{x}-\mathrm{i} \hat{y}) e^{-\mathrm{i}(m-1) \bar{\phi}_{k}}-\frac{\kappa \gamma_{0}}{2}(\hat{x}+\mathrm{i} \hat{y}) e^{-\mathrm{i}(m+1) \bar{\phi}_{k}}+\tilde{b}_{m} \hat{R}+\mathrm{i} \tilde{a}_{m} \hat{\phi}
\end{aligned}
$$

where, with $e_{0}=e^{z_{0} / 2 H}$ as before,

$$
\begin{aligned}
S_{m n} & =e_{0} \sum_{j=1}^{n} \sin m \bar{\phi}_{j} \sin \bar{\phi}_{j}=\frac{n}{2}\left(\delta_{m 1}-\delta_{m, n-1}\right) e_{0} \\
C_{m n} & =e_{0} \sum_{j=1}^{n} \cos m \bar{\phi}_{j} \cos \bar{\phi}_{j}=\frac{n}{2}\left(\delta_{m 1}+\delta_{m, n-1}\right) e_{0}
\end{aligned}
$$

and

$$
\begin{aligned}
& \tilde{a}_{m}=\sum_{j=1}^{n} \bar{a}_{j} \sin m \bar{\phi}_{j} \quad \text { where } \quad \bar{a}_{1}=0 \quad \text { and } \quad \bar{a}_{j}=\eta_{j} s_{j} c_{j}, \quad j>1 \\
& \tilde{b}_{m}=\sum_{j=1}^{n} \bar{b}_{j} \cos m \bar{\phi}_{j} \quad \text { where } \quad \bar{b}_{1}=\kappa \frac{\mu_{0}}{\bar{r}_{0}}+\sum_{j=2}^{n} \xi_{j}+2 s_{j}^{2}\left(\mu_{j} s_{j}-\xi_{j}\right) \quad \text { and } \quad \bar{b}_{j}=\eta_{j} s_{j}^{2}-\xi_{j}, \quad j>1 \\
& \tilde{c}_{m}=\sum_{j=1}^{n} \bar{c}_{j} \cos m \bar{\phi}_{j} \quad \text { where } \quad \bar{c}_{1}=\sum_{j=2}^{n} \xi_{j}-\eta_{j} c_{j}^{2} \quad \text { and } \quad \bar{c}_{j}=\eta_{j} c_{j}^{2}-\xi_{j}, \quad j>1
\end{aligned}
$$

where $\eta_{j}=2\left(\mu_{j} s_{j}+\xi_{j}\right)$ as before.

For consistency, Eqs. (3.36) and (3.37) must be independent of $k$. There are various cases to consider, depending on $m$ and $n$. When $n=2$, the only permissible mode is $m=1$. Then the exponentials involving $k$ are all equal to 1 . This results in the following $4 \times 4$ matrix from which the eigenvalues are determined:

$$
\left(\begin{array}{cccc}
0 & -P_{02} & \mathrm{i} \gamma_{0} S_{12} & \xi_{0} C_{12} \\
Q_{02} & 0 & -\gamma_{0} C_{12} & \mathrm{i} \xi_{0} S_{12} \\
0 & \kappa \xi_{0} & \mathrm{i} \tilde{a}_{1} & \tilde{c}_{1} \\
-\kappa \gamma_{0} & 0 & \tilde{b}_{1} & \mathrm{i} \tilde{a}_{1}
\end{array}\right) .
$$


But in fact $S_{12}=0$ while $C_{12}=2 e_{0}$, and moreover $\tilde{a}_{1}=0$, so the stability matrix simplifies to

$$
\left(\begin{array}{cccc}
0 & -P_{02} & 0 & \xi_{0} C_{12} \\
Q_{02} & 0 & -\gamma_{0} C_{12} & 0 \\
0 & \kappa \xi_{0} & 0 & \tilde{c}_{1} \\
-\kappa \gamma_{0} & 0 & \tilde{b}_{1} & 0
\end{array}\right) .
$$

The structure of the matrix means that eigenvalues are determined from a quadratic equation in $\sigma^{2}$.

When $n>2$ and $m=1$, we must have $\hat{y}=\mathrm{i} \hat{x}$. Then, since $P_{0 n}=Q_{0 n}$ in this case, and also $C_{1 n}=S_{1 n}=n e_{0} / 2$, Eq. (3.35) is implied by Eq. (3.34) (they are proportional to each other). Hence, only the equations for $\hat{x}, \hat{R}$ and $\hat{\phi}$ are independent, and the stability matrix reduces to

$$
\left(\begin{array}{ccc}
-\mathrm{i} P_{0 n} & \mathrm{i} \gamma_{0} C_{1 n} & \xi_{0} C_{1 n} \\
\mathrm{i} \kappa \xi_{0} & \mathrm{i} \tilde{a}_{1} & \tilde{c}_{1} \\
-\kappa \gamma_{0} & \tilde{b}_{1} & \mathrm{i} \tilde{a}_{1}
\end{array}\right) .
$$

Finally, when $n>3$ and $2 \leq m \leq[n / 2]$, both exponential terms involving $k$ vary with $k$. Hence we must have $\hat{x}=\hat{y}=0$ in this case. Note $S_{m n}=C_{m n}=0$, so Eqs. (3.34) and (3.35) drop out. We are left with a $2 \times 2$ stability matrix for each $m$ :

$$
\left(\begin{array}{cc}
\mathrm{i} \tilde{a}_{m} & \tilde{c}_{m} \\
\tilde{b}_{m} & \mathrm{i} \tilde{a}_{m}
\end{array}\right) \text {. }
$$

Thus the eigenvalues may be determined explicitly from

$$
\sigma=\mathrm{i} \tilde{a}_{m} \pm \sqrt{\tilde{b}_{m} \tilde{c}_{m}}
$$

so instability requires $\tilde{b}_{m} \tilde{c}_{m}>0$.

\subsection{Results}

A small sample of results are presented next, before providing a general summary over parameter space. First, the results for $\lambda \equiv 1 / H=0$ (the Boussinesq limit) were verified against the independent analysis of Reinaud [10], who shared his numerical code. (His code applies to general configurations of Boussinesq QG vortices, not specifically to the ring configuration considered in the present study.) All results for general values of $\kappa_{0}, z_{0}$ and $n$ agree to machine precision. Second, a nonlinear code solving Eqs. (2.12) and (2.13) (extended from $j=0$ to $n$ ) was used to verify stability/instability boundaries in a few selected cases when $\lambda>0$. For example, when $\kappa_{0}=1$ and $z_{0}=-1$, eight vortices on a ring are unstable for $\lambda=5.95$ but stable for $\lambda=5.96$, as verified by the nonlinear code. Accuracy of the simulations is monitored by checking conservation of the 'excess energy' (or vortex interaction energy)

$$
E=\sum_{k=0}^{n} \sum_{j=0, j \neq k}^{n} \kappa_{k} \kappa_{j} e^{\left(z_{k}-z_{j}\right) / 2 H} g\left(\Delta_{k j}\right) .
$$

We first illustrate how linear stability varies with the inverse scale height $\lambda=1 / H$. In Fig. 1 we consider a central vortex at height $z_{0}=-1$ and having five different strengths, $\kappa_{0}=0, \pm 2$ and \pm 4 . Negative $\kappa_{0}$ is generally destabilising (see dashed curves), with the exception of $n=2$ below $\lambda=3.75047$ (this transition value depends on $z_{0}$ ). In fact, $n=3,4$ and 5 ring vortices are entirely stable for $\kappa_{0} \geq 0$. As $n$ increases further, instability extends to positive $\kappa_{0}$, and the growth rates increase. Note, when $\kappa_{0}=0$, the central vortex is a passive particle. The instability seen for $n=2$ 
only displaces this particle without affecting the vortices on the ring. That is, two vortices on a ring are linearly stable, and this true for all $H$. On the other hand, the instabilities seen for 6-9 vortices (and beyond) affect the vortices on the ring as well. They are present even without a central vortex. At larger $\lambda$ (or smaller $H$ ), the growth rates decrease but only tend to zero as $\lambda \rightarrow \infty$. However, the largest growth rates typically occur for intermediate $\lambda$ around 2 to 3 , and only occasionally in the Boussinesq limit $\lambda=0$.
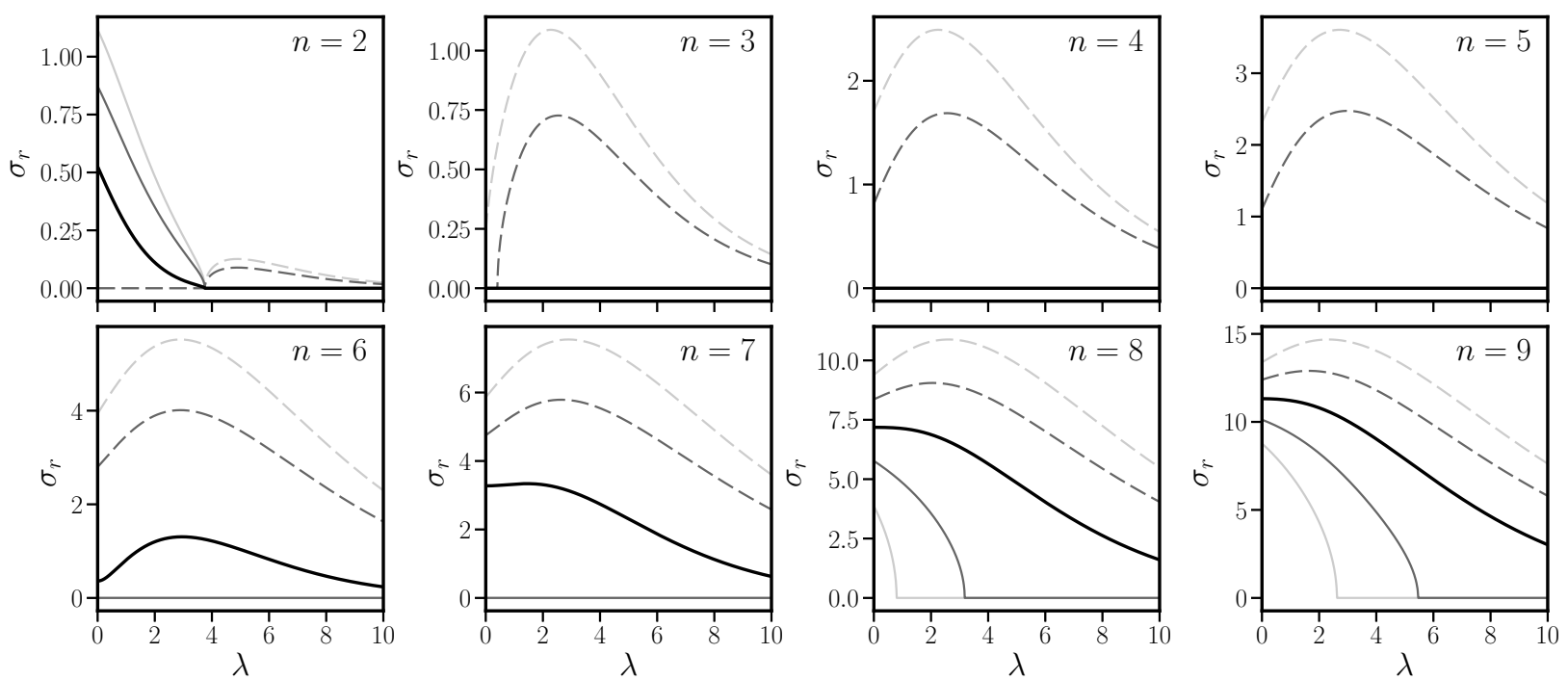

Fig. 1. Maximum growth rate $\sigma_{r}$ (the real part of $\sigma$ ) as a function of inverse scale height $\lambda=1 / H$, for $n=2$ to 9 vortices on a ring, including a central vortex. Here, the central vortex is located below the ring at $z_{0}=-1$, and five values of its strength $\kappa_{0}$ are compared: -4 (light grey, dashed line), -2 (medium grey, dashed line), 0 (black, bold line), 2 (medium grey, solid line), 4 (dark grey, solid line).

To obtain a more comprehensive picture of the stability of these vortex configurations, we next vary both the strength $\kappa_{0}$ and height $z_{0}$ of the central vortex, for a few values of the inverse scale height $\lambda$. The maximum growth rate, for $n=2$ to 9 vortices, is shown first in Fig. 2 for the Boussinesq case, $\lambda=0$. These results reproduce those in [10], although in a different figure layout. There is reflectional symmetry about $z_{0}=0$, but only for this value of $\lambda=0$. For $n=2$ and 3 , the dominant instabilities are found for $\kappa_{0}>0$, i.e. a co-rotating central vortex, and for $z_{0}=0$. The picture reverses for $n \geq 4$ vortices, though $n=4$ still has a tongue of weak instability for $\kappa_{0}>0$ (this is also true for $n=5$ for $\kappa_{0}>6.74496$ which is beyond the plot window). For $2 \leq n \leq 5$ vortices, most of the parameter plane is stable, but this changes for $n \geq 6$. Then, only a wedge of $z_{0}$ values are stable, and only for $\kappa_{0}>0$. Over the range of $\kappa_{0}$ and $z_{0}$ considered, $n=3$ vortices have the smallest growth rates, followed by $n=2$ and 4 . For $n>4$, growth rates increase with $n$.

Fig. 3 shows the maximum growth rates for $\lambda=2$. The asymmetry in $z_{0}$ is apparent, especially for small $n$ when the inter-vortex separation on the ring is relatively large, and thus more strongly influenced by the finite scale height $H$ (here $=0.5$ ). As in the Boussinesq case in Fig. $2, n=2$ and 3 vortices exhibit different behaviour, namely stronger instability for $\kappa_{0}>0$ than for $\kappa_{0}<0$, compared to $n \geq 4$ vortices. For $n=3$, the region of instability for $\kappa_{0}>0$ moves toward positive $z_{0}$, while a single region of weaker instability for $\kappa_{0}<0$ moves toward negative $z_{0}$. This also occurs for $n=4$ (and $n=5$ but the weak tongue of instability for $\kappa_{0}>0$ is beyond the plot window). Again, a qualitative change occurs for $n \geq 6$, where most of the parameter space is now unstable. Then, the regions of strongest instability and stability both move towards more negative $z_{0}$.

Figs. 4 and 5 consider yet larger inverse scale heights, $\lambda=4$ and 8 respectively. Here we see that the asymmetry in $z_{0}$ increases with $\lambda$, and growth rates decrease. For $n=3$ and $\lambda=4$, there are several tongues of instability for $\kappa_{0}>0$, but the upper ones contain only very weak instabilities. The region of instability for $n=2$ extends to larger positive $z_{0}$, and becomes almost independent of $\kappa_{0}$ in that region. For $n=3$ to 5 vortices, the instability region in the lower left corner extends over 

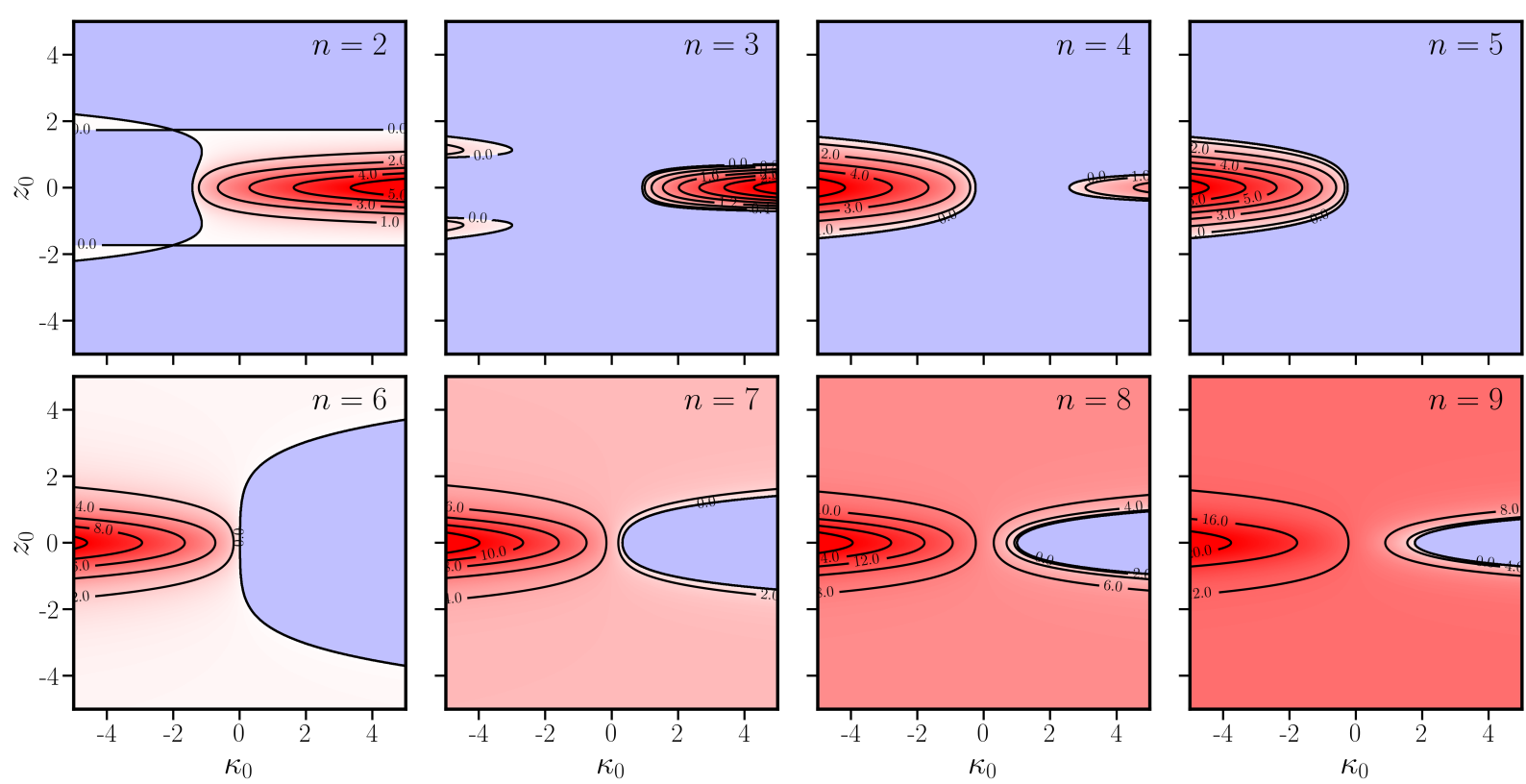

Fig. 2. Maximum growth rate as a function of both the strength $\kappa_{0}$ and the height $z_{0}$ of the central vortex, for an inverse scale height $\lambda=0$ (the Boussinesq case studied in [10]). Here $n=2$ to 9 vortices are considered. Light blue regions are (neutrally) stable.
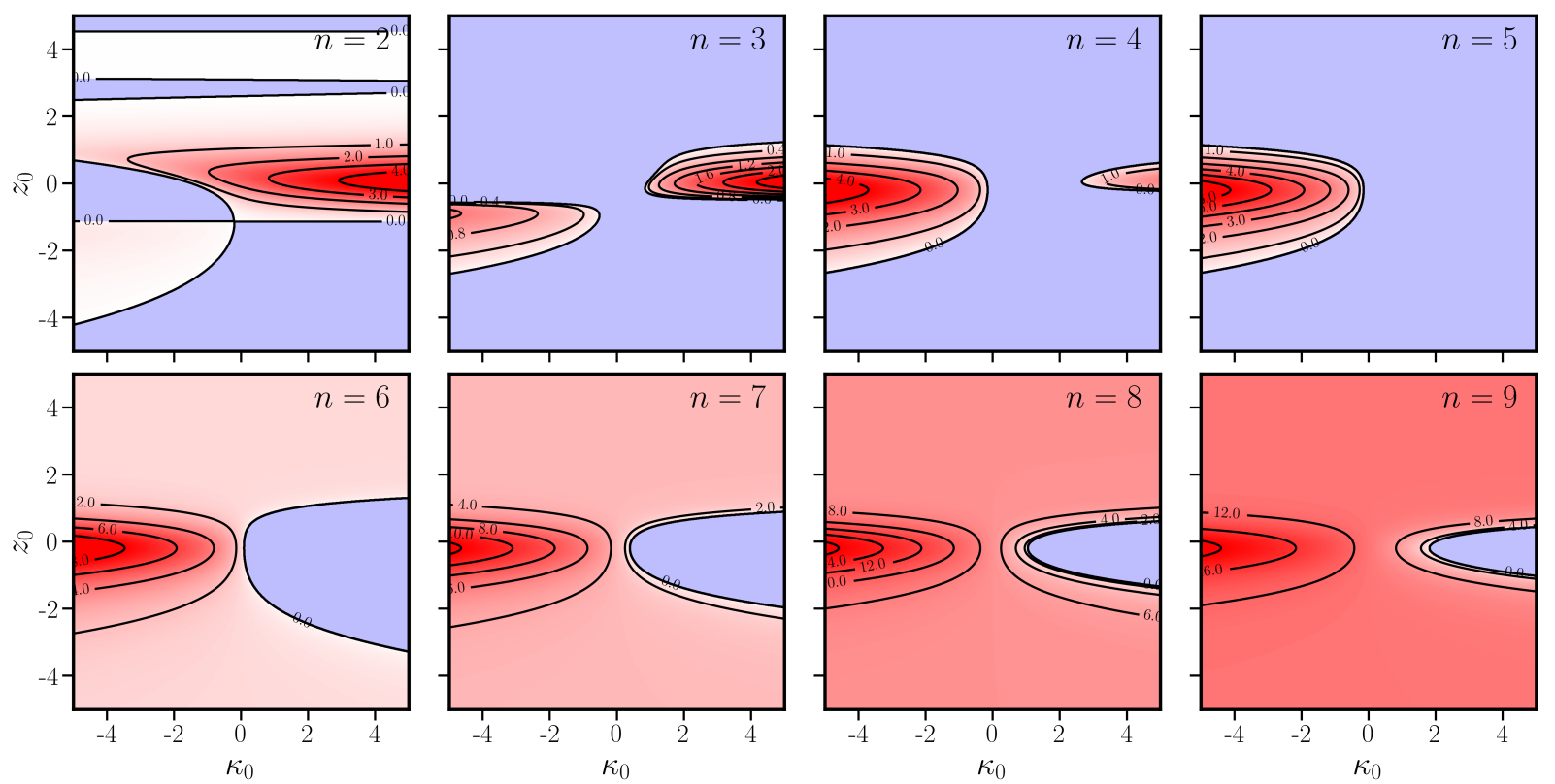

Fig. 3. Maximum growth rate as a function of both the strength $\kappa_{0}$ and the height $z_{0}$ of the central vortex, for an inverse scale height $\lambda=2$. The format is the same as in Fig. 2.

an increasing range of negative $z_{0}$, while the tongues of instability occurring for $\kappa_{0}>0$ when $n=3$ and 4 (as well as $n=5$ for $\kappa_{0}>8.849$ beyond the plot window) narrow toward $z_{0}=0$. Finally, both the stable and most unstable regions for $n \geq 6$ become increasingly confined to $z_{0}<0$.

Taken together, the above results show that $n=5$ vortices on a ring are stable over a greater portion of parameter space than any other number of vortices. In particular, five vortices are stable without a central vortex, or with any co-rotating central vortex, regardless of its position above 

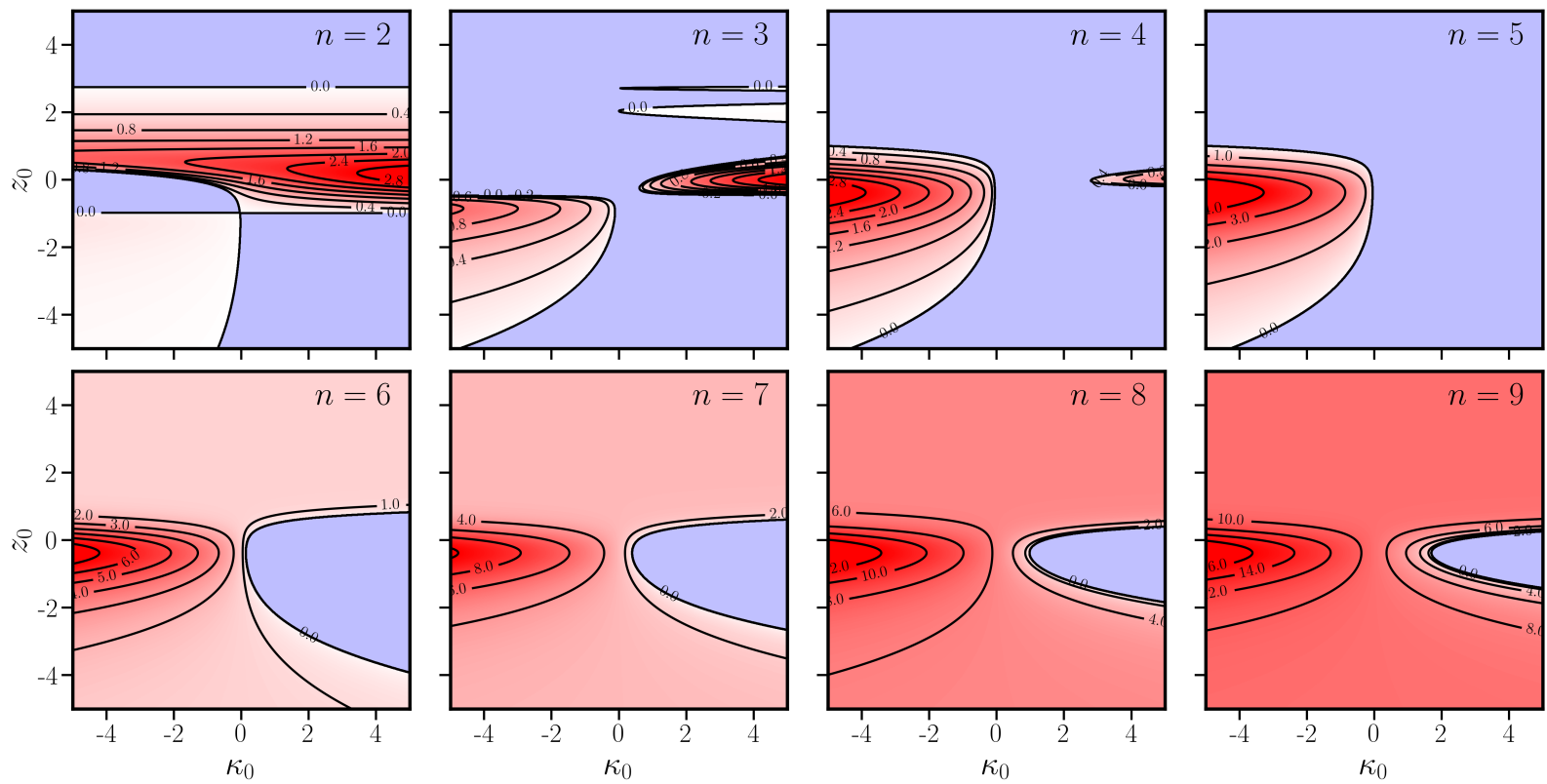

Fig. 4. Maximum growth rate as a function of both the strength $\kappa_{0}$ and the height $z_{0}$ of the central vortex, for an inverse scale height $\lambda=4$. The format is the same as in Fig. 2.
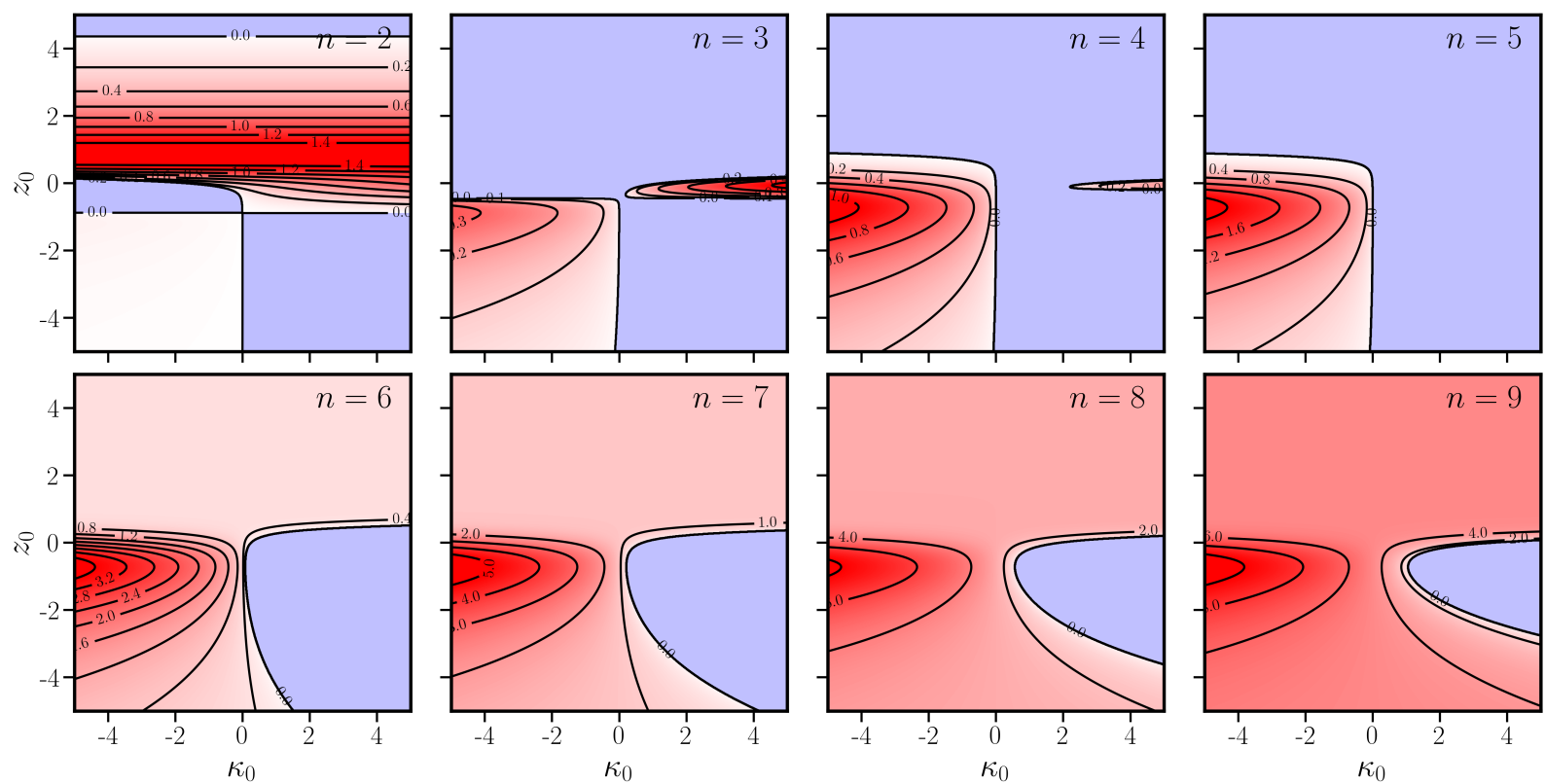

Fig. 5. Maximum growth rate as a function of both the strength $\kappa_{0}$ and the height $z_{0}$ of the central vortex, for an inverse scale height $\lambda=8$. The format is the same as in Fig. 2.

or below the ring. The domain of linear stability qualitatively changes between $n \leq 5$ vortices and $n \geq 6$ vortices. For $n \leq 5$, there is always a narrow tongue of instability for $\kappa_{0}>0$ near and just above $z_{0}=0$ (with the exception of $n=2$ which has a wide domain of instability). For $n \geq 6$, a region of stability opens up for $\kappa_{0}>0$ near $z_{0}=0$. Significantly, depending on parameters, any number of vortices can exist in a stable configuration. For $n \geq 6$, stable configurations exist with a co-rotating central vortex that is located in the plane of the ring and a little below it. This region 

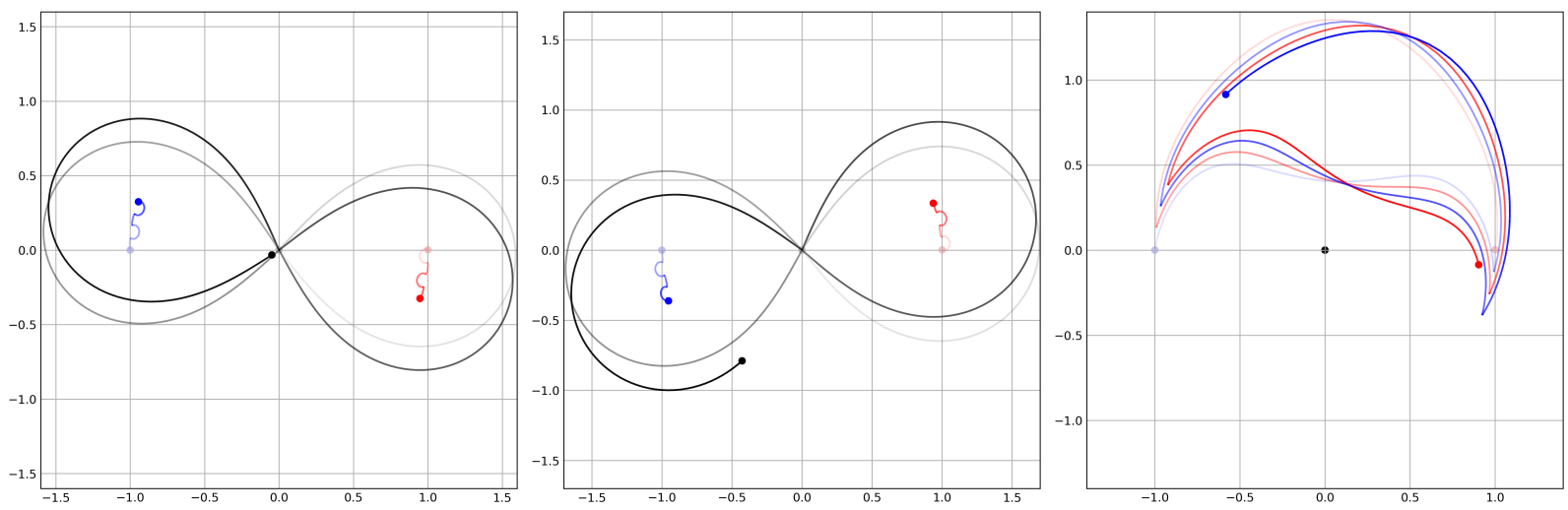

Fig. 6. Evolution of two vortices $(n=2)$ initially on a ring with a central vortex, for an inverse scale height $\lambda=4$; the left panel shows $z_{0}=1$ and $\kappa_{0}=2$ for $0 \leq t \leq 50$, the middle shows $z_{0}=1$ and $\kappa_{0}=-2$ for $0<t<50$, and the right shows $z_{0}=-2$ and $\kappa_{0}=-2$ for $0 \leq t<400$. Each vortex has a different colour, and the trajectory fades back in time (the initial positions are faded circles, and the final state are dark circles). Initially, the vortex on the right is displaced by $0.1^{\circ}$ on the unit circle. The trajectories are shown in a frame of reference rotating at the equilibrium angular velocity.

of stability shrinks with increasing $n$. In particular, an increasingly strong central vortex is required to ensure stability over a narrowing gap in $z_{0}$.

\section{NONLINEAR DYNAMICS}

We next examine the nonlinear evolution of a small sample of unstable initial conditions. The numerical code (in python) solves Eqs. (2.12) and (2.13), in a frame of reference rotating at the equilibrium angular velocity $\Omega$. The code conserves excess energy $E$ (3.48) to better than one part in $10^{8}$ by using an adaptive time step to resolve the rapid rotation of close pairs of vortices, closely similar to that described in [5].

Fig. 6 begins with three different cases for $n=2$. Each case has a central vortex and a fixed inverse scale height $\lambda=4$. The left and middle panels both have the height of the central vortex $z_{0}=1$ above the plane of the ring, but differ only in the strength of the central vortex, with $\kappa_{0}=2$ in the left panel and $\kappa_{0}=-2$ in the middle panel. The trajectories are similar but precess in opposite directions. The middle vortex moves far from its initial position, first circling the vortex on the right, then the one on the left (and this continues to much longer times than shown here). Both these cases are lying in the region of relatively large growth rates in Fig. 4 (top left panel). The case on right has $z_{0}=-2$ and $\kappa_{0}=-2$, and lies in the region of weaker growth rates. Here, the evolution is very different to the previous two cases: the central vortex hardly moves, while the two vortices on the ring mainly move closer together for a period, then further apart, and so on, while also precessing clockwise.

Moving on, Fig. 7 shows three different cases for $n=3$ vortices initially on the ring. All cases again include a central vortex and take $\lambda=4$. The left panel, for $z_{0}=0$ and $\kappa_{0}=2$, is taken from the region of strongest instabilities in Fig. 4 (top middle panel). In this case, all four vortices move in an erratic manner, exhibiting strong radial excursions. The middle panel, for $z_{0}=2$ and $\kappa_{0}=4$, is taken from the region of weak instabilities centred on $z_{0}=2$ and having $\kappa_{0}>0$ in Fig. 4. In this case, the central vortex slowly spirals out then back in (this repeats at later times beyond those shown here). The vortices on the ring hardly move. Finally, the right panel, for $z_{0}=-1$ and $\kappa_{0}=-2$, is taken from the region of moderate instabilities in the lower left portion of the $n=3$ panel in Fig. 4. This time the central vortex hardly moves, while the vortices initially on the ring move in a complicated manner. The vortex on the right returns close to its initial position by $t=50$ while the other two vortices approximately exchange positions.

Fig. 8 next shows three cases for $n=4$ vortices initially on the ring, with a central vortex and for $\lambda=4$. The case in the left panel, for $z_{0}=0$ and $\kappa_{0}=4$, lies in the small and narrow unstable region for $\kappa_{0}>0$ in Fig. 4 (for $n=4$ ). All vortices move somewhat erratically, yet the vortices on the ring stay with a narrow range of radii while drifting, on average, counter-clockwise. The central 

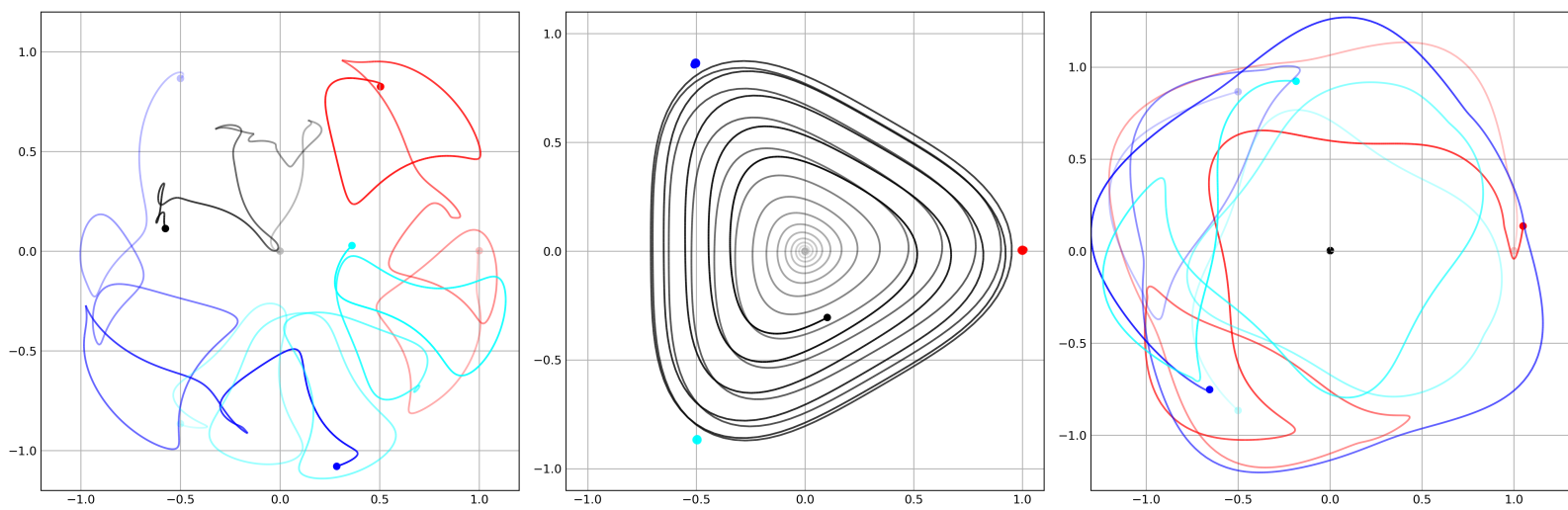

Fig. 7. Evolution of three vortices $(n=3)$ initially on a ring with a central vortex, for an inverse scale height $\lambda=4$; the left panel shows $z_{0}=0$ and $\kappa_{0}=2$ for $0 \leq t \leq 25$, the middle shows $z_{0}=2$ and $\kappa_{0}=4$ for $0 \leq t \leq 500$, and the right shows $z_{0}=-1$ and $\kappa_{0}=-2$ for $0 \leq t \leq 50$. The layout is the same as described in Fig. 6 .
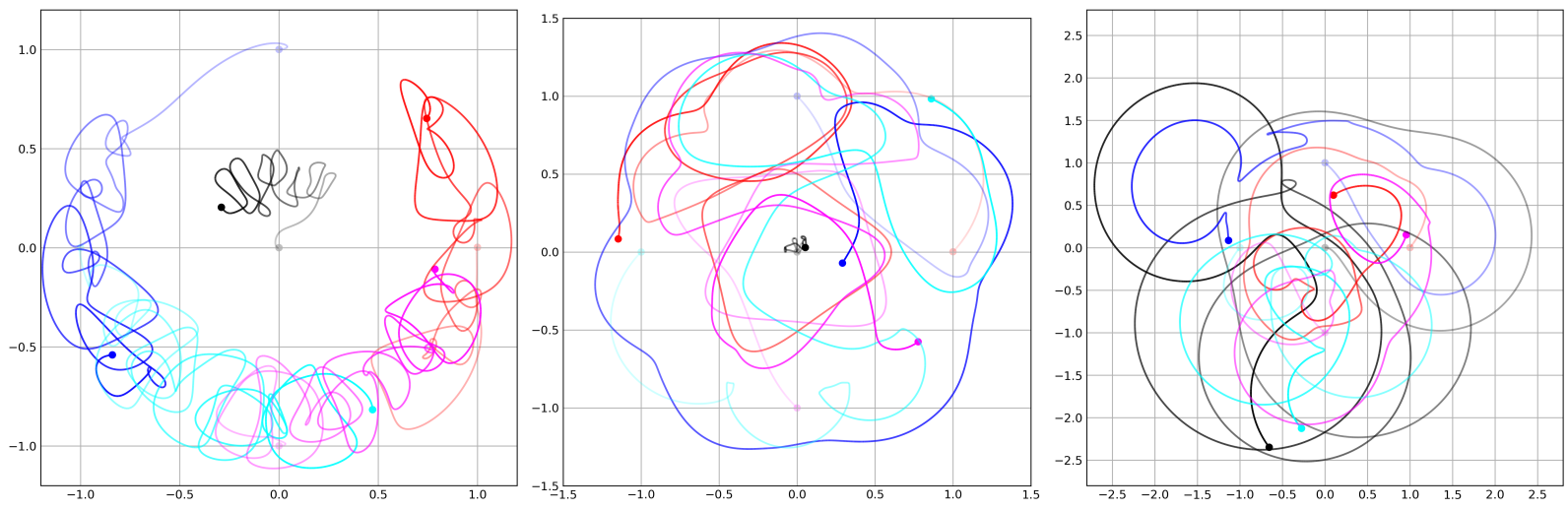

Fig. 8. Evolution of four vortices $(n=4)$ initially on a ring with a central vortex, for an inverse scale height $\lambda=4$; the left panel shows $z_{0}=0$ and $\kappa_{0}=4$ for $0 \leq t \leq 25$, the middle shows $z_{0}=-1$ and $\kappa_{0}=-2$ for $0<t<25$, and the right shows $z_{0}=0.5$ and $\kappa_{0}=-4$ for $0 \leq t \leq 25$. The layout is the same as described in Fig. 6 .

vortex also remains near the centre but exhibits a complicated trajectory. The cases in the middle and right panels are taken from the larger region of instability for $\kappa_{0}<0$ in Fig. 4. The middle case, for $z_{0}=-1$ and $\kappa_{0}=-2$, exhibits strong radial and azimuthal excursions of the vortices initially on the ring. Each such vortex moves on a qualitatively different trajectory, e.g. one (in magenta) wraps around close to the origin while another (in blue) stays relatively far from the origin until close to the end at $t=25$. Remarkably, the initially central vortex remains close to the origin. The case on the right, for $z_{0}=0.5$ and $\kappa_{0}=-4$, exhibits particularly large radial excursions; here the central vortex moves far from the origin. All vortices move erratically. Note that the central vortex briefly pairs with a different vortex, and the two move along nearly circular arcs.

We skip the case $n=5$ and focus on $n=6$, for which three cases are shown in Fig. 9. As before, these include a central vortex and are for $\lambda=4$. The case in the left panel, for $z_{0}=2$ and $\kappa_{0}=2$, lies in the upper right part of the unstable region, above the stable region, in Fig. 4 (for $n=6$ ). Here, growth rates of the unstable modes are weak. The evolution is highly regular, and in fact commonly occurs throughout the region of weak instability, except in the quadrant $z_{0}<0$ and $\kappa_{0}<0$. The vortices initially on the ring move into a staggered configuration on two rings with three equallyspaced vortices on each. They then move back to a ring configuration and to an opposite staggered configuration. Sometimes, the vortices return to the same staggered configuration (not shown in this example). This oscillation was examined previously by [13] in the two-layer quasi-geostrophic model (and without a central vortex), but it is expected to be similar here. The case in the middle 

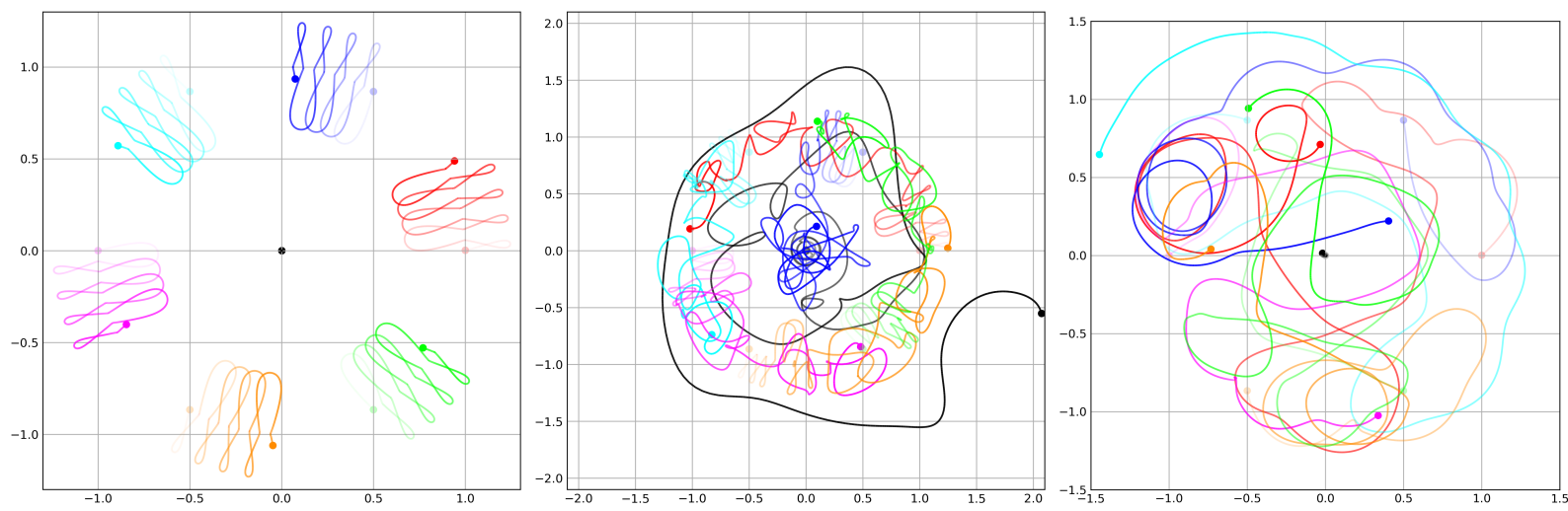

Fig. 9. Evolution of six vortices $(n=6)$ initially on a ring with a central vortex, for an inverse scale height $\lambda=4$; the left panel shows $z_{0}=2$ and $\kappa_{0}=2$ for $0 \leq t \leq 100$, the middle shows $z_{0}=1$ and $\kappa_{0}=-2$ for $0 \leq t \leq 100$, and the right shows $z_{0}=-1$ and $\kappa_{0}=-2$ for $0 \leq t \leq 10$. The layout is the same as described in Fig. 6 .

panel, for $z_{0}=1$ and $\kappa_{0}=-2$, lies just above the stronger tongue of instability in Fig. 4. At early times, the evolution is similar to the first case, but this breaks down into erratic behaviour, with the central vortex moving far away from the others (it moves even further out at later times, not shown). One of the vortices on the ring (in blue) moves toward the origin, while the five remaining ring vortices remain in a more limited range of radii. The case in the right panel, for $z_{0}=-1$ and $\kappa_{0}=-2$, lies in the region of stronger instability, and after a relatively short time the vortices begin to move erratically. Notably, the central vortex remains close to the origin. In the parameter space below this region of stronger instability, the evolution is similar but somewhat more regular, with one of the ring vortices moving above the central vortex (which stays nearly fixed) for a period, only to be replaced by another ring vortex and so on (not shown).

Regarding other values of $n$, the behaviour of $n=5$ ring vortices (when linearly unstable) is qualitatively similar to that just seen in the right panel of Fig. 9 or in the middle panel of Fig. 8. Only erratic motion is ever observed (based on a wide sampling of parameter space). However, there are extensive regions of parameter space where $n=5$ ring vortices are stable, even for finiteamplitude perturbations. For $n \geq 6$, instability is much more widespread. Instabilities for $n=7$ ring vortices (and likely all odd $n>7$ ) also exhibit erratic motion. The case of $n=8$ is similar to $n=6$ : staggered configurations occur in regions of weak instability, here close to the margin of stability (e.g. $z_{0}=-1$ and $\kappa_{0}=1.5$ when $\lambda=4$ ). Elsewhere, erratic motion occurs.

While all the examples here used an inverse scale height $\lambda=4$, qualitatively similar results are found for other $\lambda$. In particular the oscillatory staggered configurations for $n=6$ and $n=8$ also occur in the Boussinesq limit $\lambda \rightarrow 0$, examined by [10] and more recently in [11] (this issue). Staggered configurations require even $n$.

\section{CONCLUSIONS}

This paper has discussed the classical problem first studied by J.J. Thomson [14] concerning the stability of a regular array of equal point vortices arranged on a ring. Here, the problem has been extended to point vortices in a three-dimensional compressible atmosphere, under the quasigeostrophic approximation. The latter yields equations that are closely similar to those studied by [14], with the only difference being the Green function controlling the vortex interactions. A central point vortex at any height above or below the ring is also included.

The incompressible, Boussinesq limit was recently studied by [10], who comprehensively mapped the linear stability and further generalised the problem to finite-volume vortices. Like in [10], it appears that $n=5$ vortices on the ring exhibit the widest range of stability in parameter space, closely followed by $n=4$, even in a compressible atmosphere. The main effect of compressibility is to shorten the interaction range: the density scale height $H$ acts as a screening length, and when $H$ is small compared with the spacing of vortices on the ring, interactions weaken exponentially. 

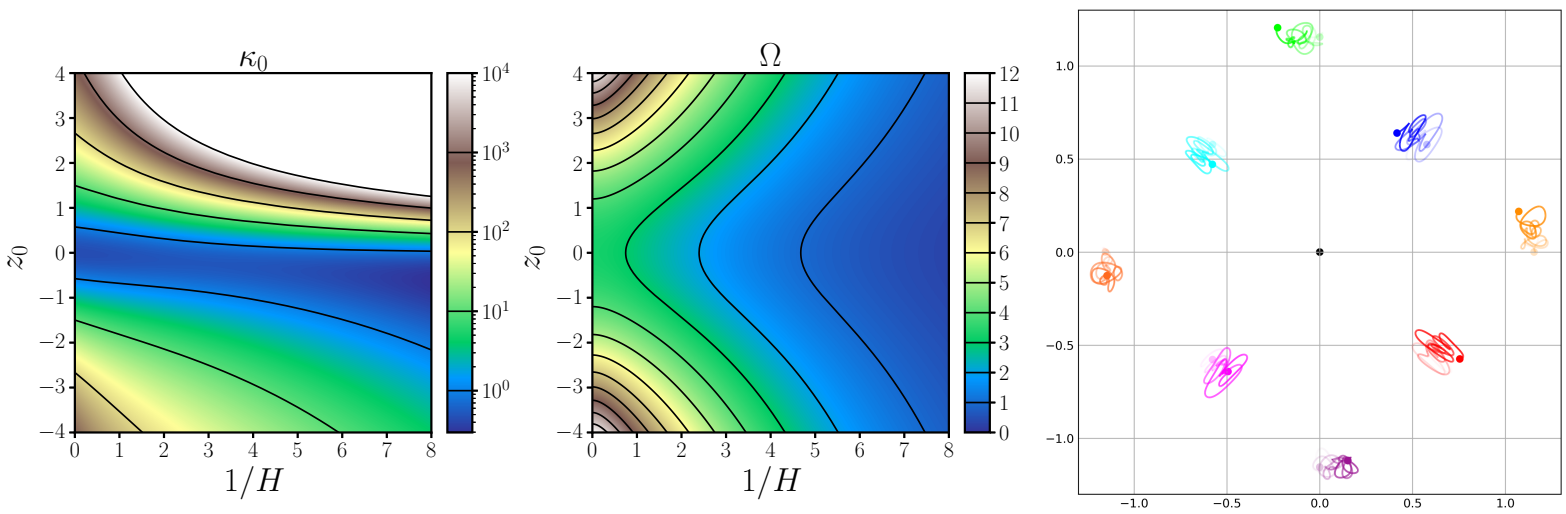

Fig. 10. Left and middle panels: equilibrium values of the central vortex strength $\kappa_{0}$ and system rotation rate $\Omega$ versus the inverse scale height $\lambda=1 / H$ and the height $z_{0}$ of the central vortex, for $n=8$ vortices staggered on two concentric rings $r=R_{a}$ and $R_{b}$. The squared radius ratio $\left(R_{a} / R_{b}\right)^{2}=0.5$, while the circulation ratio $\kappa_{a} / \kappa_{b}=1$. Note: $\left(R_{a}^{2}+R_{b}^{2}\right) / 2=1$. Right panel: evolution over $0 \leq t \leq 50$ of a configuration with $\lambda=8$ and $z_{0}=-1$, where one of the vortices on the inner ring is displaced by $\overline{5^{\circ}}$ in azimuthal angle. The equilibrium configuration in this case has $\kappa_{0}=0.346729$ and $\Omega=0.390863$ (to six decimal points). The vortices are viewed in a frame of reference rotating at the rate $\Omega$.

This has the effect of reducing growth rates and shifting the stable regions of parameter space. Whereas stability in the Boussinesq limit $H \rightarrow \infty$ depends only on the magnitude of the central vortex height $\left|z_{0}\right|$, decreasing $H$ leads to an asymmetry in $z_{0}$, with $n=2$ and $n>5$ vortices more unstable for positive $z_{0}$, and the opposite for $3 \leq n \leq 5$ vortices.

The nonlinear evolution of unstable configurations reveals instances of regular or oscillatory behaviour, predominantly for $n=2,3$ and even $n \geq 6$, for which vortices can move in and out of staggered configurations, as seen previously in [13] in a different but related flow model. In fact, symmetry considerations alone indicate that steadily-rotating staggered configurations exist for all even $n$, depending on the strength of the central vortex $\kappa_{0}$. Moreover, the strengths of the vortices on each ring can be different, adding another degree of freedom. Fig. 10 shows examples of these equilibria when the squared radius ratio of the two rings is 0.5 and the circulation ratio is 1. Notably, the rotation rate $\Omega$ is symmetric in $z_{0}$, while the central vortex strength $\kappa_{0}$ is not. The smallest values of $\kappa_{0}$ are found for $z_{0}<0$, but $\kappa_{0}$ remains positive across parameter space. This figure also shows that stable configurations exist: the case on the right (for $\lambda=8$ and $z_{0}=-1$ ) is initialised with a large displacement of one of the vortices, yet the vortices remain close to their original positions. Further results for these staggered configurations in the Boussinesq limit $(\lambda=0)$, including linear stability, can be found in [11] (this issue).

The results in this paper may be relevant to the vortex arrays recently observed around both of Jupiter's poles [1]. A staggered set of 8 vortices, possibly with a central vortex, was observed at the north pole, while 5 vortices with a central vortex was observed at the south pole. The present paper indicates that both of these configurations may be long-lived if they lie in the stable region of parameter space. However, estimating parameters is difficult, and the model studied in this paper may be too idealised. The point vortex approximation only captures the leading-order interaction of finite-volume vortices, and the quasi-geostrophic approximation may be too idealised. Nevertheless, the present model is perhaps the simplest model containing the key features likely to be found in polar regions of rapidly-rotating and strongly-stratified planetary atmospheres: the model is threedimensional, nonlinear, and includes the effect of strongly decreasing density with height. A step toward greater realism would be to follow [10] and consider finite volume vortices.

\section{CONFLICT OF INTEREST}

The author declares that he has no conflicts of interest.

REGULAR AND CHAOTIC DYNAMICS Vol. 00 No. $0 \quad 0000$ 


\section{ACKNOWLEDGMENTS}

I wish to thank Jean Reinaud for helpful discussions about this research and for his comments on a draft of this paper. I am grateful to a referee for pointing out an error in the stability analysis which fortunately had no impact on the results.

\section{REFERENCES}

1. Adriani, A., Mura, A., Orton, G., Hansen, C., Altieri, F., Moriconi, M. L., Rogers, J., Eichstdt, G., Momary, T., Ingersoll, A. P., Filacchione, G., Sindoni, G., Tabataba-Vakili, F., Dinelli, B. M., Fabiano, F., Bolton, S.J., Connerney, J.E.P., Atreya, S. K., Lunine, J.I., Tosi, F., Migliorini, A., Grassi, D., Piccioni, G., Noschese, R., Cicchetti, A., Plainaki, C., Olivieri, A., ONeill, M.E., Turrini, D., Stefani, S., Sordini, R. and Amoroso, M., Cluster of cyclones encircling Jupiter's poles, Nature, 2018, vol. 555, pp. 216-219.

2. Andrews, D. G., Holton, J. R., and Leovy, C. B., Middle Atmosphere Dynamics, Academic Press, 1987.

3. Dritschel, D. G. and Saravanan, R., Three-dimensional quasi-geostrophic contour dynamics, with an application to stratospheric vortex dynamics. Quart. J. Roy. Meteorol. Soc., 1994, vol.120, pp. 12671297.

4. Dritschel, D. G. and Boatto, S., The motion of point vortices on closed surfaces, Proc. Roy. Soc. A, 2015, vol. 471, pp. 20140890.

5. Dritschel, D. G., Equilibria and stability of four point vortices on a sphere, Proc. Roy. Soc. A, 2020, vol. 476, pp. 20200344.

6. Kirchhoff, G., Vorlesungen über mathematische Physik. Mechanik, Leipzig: Teubner, 1876.

7. Kurakin, L. G. and Ostrovskaya, I. V., On Stability of Thomson's Vortex $N$-gon in the Geostrophic Model of the Point Bessel Vortices, Regular and Chaotic Dynamics, 2017, vol. 22(7), pp. 865-879.

8. Morikawa, G. K. and Svenson, E. V., Interacting Motion of Rectilinear Geostrophic Vortices, Physics of Fluids, 1971, vol. 14(6), pp. 1058-1073.

9. Reinaud, J.N., Dritschel, D. G. and Koudella, C.R., The shape of the vortices in quasi-geostrophic turbulence, J. Fluid Mech., 2003, vol. 474, pp. 175-191.

10. Reinaud, J. N., Three-dimensional quasi-geostrophic vortex equilibria with $m$-fold symmetry, J. Fluid Mech., 2019, vol. 863, pp. 32-59.

11. Reinaud, J. N., Three-dimensional quasi-geostrophic staggered vortex arrays, Regular and Chaotic Dynamics, 2021, vol. xxx, pp. xxx-xxx.

12. Scott, R. K. and Dritschel, D. G., Quasi-geostrophic vortices in compressible atmospheres, J. Fluid Mech., 2005, vol. 530, pp. 305-325.

13. Sokolovskiy, M. A., Koshel, K. V., Dritschel, D. G. and Reinaud, J. N., $N$-symmetric interaction of $N$ hetons. Part I: Analysis of the case $N=2$. Physics of Fluids, 2020, vol. 32(9), pp. 096601.

14. Thomson, J. J., A Treatise on the Motion of Vortex Rings, London: MacMillan, 1883, pp. 94-108.

15. Vallis, G.K., Atmospheric and Oceanic Fluid Dynamics, Cambridge: Cambridge University Press, 2006. 